\title{
MEK/Aurora Kinase Inhibitor BI 847325
}

National Cancer Institute

\section{Source}

National Cancer Institute. MEK/Aurora Kinase Inhibitor BI 847325. NCI Thesaurus. Code C106126.

An orally available dual inhibitor of mitogen-activated protein kinase kinase (MEK) and Aurora kinases, with potential antineoplastic activity. Upon oral administration, MEK/Aurora kinase inhibitor BI 847325 selectively binds to and inhibits the activity of MEK, which both prevents the activation of MEK-dependent effector proteins and inhibits growth factor-mediated cell signaling. BI 847325 also binds to and inhibits the activity of the Aurora kinases A, B and C which may disrupt the assembly of the mitotic spindle apparatus, prevent chromosome segregation, and inhibit both cellular division and proliferation in Aurora kinase-overexpressing tumor cells. Altogether, this leads to the inhibition of cell proliferation and tumor growth as well as the induction of tumor regression. MEK, a dual-specificity threonine/tyrosine kinase that plays a key role in the activation of the RAS/RAF/MEK/ERK signaling pathway, is frequently upregulated in a variety of tumor cell types. Aurora kinases are serine-threonine kinases that play essential roles in mitotic checkpoint control and are overexpressed by a wide variety of cancer cell types. 